\title{
Archéopages
}

Archéopages

Archéologie et société

40 | 04-07/2014

Villages

\section{Le village lorrain du Moyen Âge à nos jours. Architecture et organisation spatiale des maisons}

\section{Franck Gérard}

\section{(2) OpenEdition}

12 Journals

Édition électronique

URL : https://journals.openedition.org/archeopages/633

DOI : 10.4000/archeopages.633

ISSN : 2269-9872

\section{Éditeur}

INRAP - Institut national de recherches archéologiques préventives

\section{Édition imprimée}

Date de publication : 1 juin 2015

Pagination : 104-119

ISSN : 1622-8545

\section{Référence électronique}

Franck Gérard, «Le village lorrain du Moyen Âge à nos jours. Architecture et organisation spatiale des maisons », Archéopages [En ligne], 40 | 04-07/2014, mis en ligne le 01 juillet 2016, consulté le 22 janvier 2022. URL : http://journals.openedition.org/archeopages/633 ; DOI : https://doi.org/10.4000/ archeopages. 633 


\section{Le village lorrain du Moyen Âge à nos jours Architecture et organisation spatiale des maisons}

Franck Gérard Inrap

1. Ce conflit oppose entre 1630 et 1660 les Habsbourg d'Espagne et le Saint Empire romain germanique aux États allemands protestants auxquels s'allient notamment les Provinces Unies et la France. Au cours de cette période, la Lorraine perd la moitié de ses habitants.

2. De nombreux villages disparaissent entre le XIII ${ }^{e}$ siècle et le $\mathrm{XVII}{ }^{\mathrm{e}}$ siècle en raison de problèmes économiques, démographiques ou politiques; on parle alors de villages disparus ou de villages désertés.
Jusqu'à l'essor de l'archéologie préventive dans les années 1970, le village et la maison demeuraient presque exclusivement un sujet d'étude réservé aux géographes. En Lorraine, Claude Gérard et Jean Peltre ont considérablement participé à la mise en valeur de ce patrimoine rural par le biais de nombreuses productions bibliographiques (Gérard, Peltre, 1979 ; Gérard, 1982, 1990 ; Peltre, 1981, 1989). De fait, l'étude de ce terroir portait de façon exclusive sur des documents d'archives et sur ce que le village contemporain laissait encore percevoir de ses origines anciennes. Létude historique, sociologique (spatiale) et architecturale de ce qui constitue la clef de voûte de la ruralité, à savoir le village et la maison, ne pouvait par conséquent que difficilement remonter au-delà du XVII ${ }^{\mathrm{e}}$ siècle, période au cours de laquelle s'est déroulée la dévastatrice guerre de Trente Ans ${ }^{1}$ et où de nombreux villages se sont vu tout simplement rayés de la carte.

La multiplication des fouilles archéologiques de grande surface ainsi que des études plus ponctuelles menées au cœur des villages actuels ont dès lors permis aux archéologues d'étendre les connaissances au-delà de cette barrière historique en soulevant tout un pan presque inconnu de l'histoire du village et de la maison rurale, qu'ils aient aujourd'hui disparu ${ }^{2}$ ou soient encore en cours de remodelage... Reste à définir ce que l'archéologue entend par la notion de village dont la définition, ou tout du moins l'intérêt qu'on y porte, diffère selon qu'on se place du côté de l'historien, du géographe, de l'ethnologue, de l'architecte ou de l'archéologue. Si les uns n'aborderont le village qu'à partir des notions purement historiques ou matérielles, les autres considéreront le village dans une approche plus globale associant le bâti, le terroir, mais également le vivant. L'archéologue dispose du privilège d'associer par sa discipline l'ensemble de ces réflexions, qu'elles relèvent de l'Homme ou de son milieu, naturel ou culturel. C'est dans cette approche que la maison rurale devient, même lorsqu'elle ne subsiste plus que sous la forme de vestiges, un signe de reconnaissance sociale, culturelle, professionnelle et économique. Son évolution architecturale, son organisation interne et son implantation dans le terroir sont autant d'indices qui contribueront à définir le village, à l'appréhender et à en comprendre la complexité [ill. 1].

L'occupation du sol et l'architecture des maisons de la fin de l'Antiquité au VIII ${ }^{\mathrm{e}}$ siècle

Elle s'opère sur le plan spatial selon les mêmes modalités qu'au cours de l'Antiquité. Les domaines et autres habitats dispersés sont d'ailleurs très souvent construits sur l'emplacement ou à proximité immédiate des anciennes villae. Jusqu'au XII ${ }^{\mathrm{e}}$ siècle, les constructions les plus fréquentes dans nos régions sont constituées de matériaux périssables. Ces techniques diffèrent peu ou pas de celles mises en œuvre progressivement à partir du IV ${ }^{\mathrm{e}}$ siècle. Elles reposent sur un système de poteaux plantés dans le sol qui servent de support à l'élévation des bâtiments. Pour pallier les problèmes d'entretien (pourrissement des poteaux au ras du sol), cette technique de construction va être progressivement remplacée par des méthodes architecturales permettant d'isoler les maisons du sol. 
À Mexy, ce mode de construction sera utilisé de façon exceptionnelle dès les $\mathrm{VII}^{\mathrm{e}}$-VIIII ${ }^{\mathrm{e}}$ siècles. Le site s'articule autour d'un ensemble empierré interprété comme l'emplacement primitif d'un édifice en bois reposant sur un soubassement de blocs calcaires de type solin. Il occupe une superficie originelle de $60 \mathrm{~m}^{2}$ environ, soit un quadrilatère de $10 \mathrm{~m}$ de long et $6 \mathrm{~m}$ de large. Les blocs ont été éparpillés a posteriori par les labours et l'érosion sur une surface de près de $500 \mathrm{~m}^{2}$ [ill. 2]. Leur répartition spatiale permet d'envisager, grâce à au moins deux alignements plus ou moins conservés, un système de soubassement de pierres supportant des sablières basses interrompues ponctuellement par des poteaux verticaux, d'angle ou de liaison, reposant eux-mêmes sur des dés de pierre. Le caractère fortement oblitéré de cet ensemble ne permet pas d'être affirmatif quant à cette hypothèse. Aussi est-il possible de songer à un système moins complexe de supports continus de sablières basses (composé de blocs sommairement disposés les uns à côté des autres) faisant ainsi bénéficier la maison d'un vide sanitaire ajouré de quelques dizaines de centimètres de hauteur. L'existence de planchers est bien connue dans les structures excavées du premier Moyen Âge grâce à la fouille. Cette technique assurerait un double avantage : celui d'isoler les matériaux périssables de l'humidité et celui de faciliter la circulation de l'eau sous l'édifice comme l'atteste un fossé qui traverse la zone de part en part. De nombreux exemples de ce type ont été rencontrés aux Pays-Bas, parfois dès le $\mathrm{III}^{\mathrm{e}}$ siècle de notre ère (Hoogeloon dans le Brabant-Septentrional) (Zimmermann, 1998). Ici, chaque support de poteau est constitué d'un agglomérat désorganisé de petits blocs posés sur le sol. D'autres exemples, plus tardifs et contemporains du site de Mexy, datés de la "période des migrations » (haut Moyen Âge dans nos régions), ont été recensés à Rollwitz dans les arrondissements d'Uecker-Randow et de Mecklenburg-Vorpommern en Allemagne. Ces exemples combinent à la fois des agglomérats de petites pierres et des blocs uniques plus conséquents posés sur le sol et soutenant des poteaux verticaux. Dans tous ces cas, les poteaux porteurs ne reposent pas directement sur une sablière mais sur des dés en pierre. Les sites de Lucy, de Yutz et de Vitry-sur-Orne (Gérard, Blaising, 2007) en Moselle, ainsi que ceux de Chaillon et de Dieue-sur-Meuse dans le département voisin, montrent que ce principe architectural ne se développe réellement en Lorraine qu'à partir des XII ${ }^{\mathrm{e}}$ et XIII ${ }^{\mathrm{e}}$ siècles. Le site de Mexy, installé sur le Pays haut lorrain non loin des frontières belges et allemandes, se détache par conséquent nettement des « règles » architecturales établies dans la Lorraine des vallées en adoptant des méthodes plus en vogue dans les pays du Nord.
Les modèles d'organisation spatiale et l'évolution architecturale du viII ${ }^{\mathrm{e}}$ siècle au XII ${ }^{\mathrm{e}}$ siècle

L'habitat rural ne semble s'éloigner de l'héritage antique qu'entre les VIII ${ }^{\mathrm{e}}$ et $\mathrm{IX}^{\mathrm{e}}$ siècles avec l'apparition d'un habitat groupé constitué d'unités indépendantes organisées, comme à Vitry-surOrne, Demange-aux-Eaux et Chaillon, ou dispersées comme à Yutz (Blaising, 2007), où cette genèse du village se met en place dès la seconde moitié du $\mathrm{IX}^{\mathrm{e}}$ siècle. L'habitat se caractérise par un groupement lâche de huit à dix fermes composées chacune de trois à cinq bâtiments et disposant toutes d'un puits et d'un système d'adduction d'eau. Ces fermes se répartissent dans des quartiers de culture dont la structure parcellaire semble l'héritière des axes de circulation antiques. Un seul noyau, localisé à l'emplacement du village récent de Haute-Yutz, présente un aspect plus dense. Il constituera dès le XII ${ }^{\mathrm{e}}$ siècle le cœur du village où se regrouperont toutes les fermes quand, dans le même temps, chaque quartier de culture précédemment bâti sera doté d'un parcellaire laniéré. Le cas de Yutz présente ainsi la particularité de disposer d'un système agraire dans lequel on note un important décalage entre la création des quartiers de culture et leur laniérage.

À Vitry-sur-Orne, ce modèle groupé se met en place en deux temps [ill. 3] : dès la première moitié du VIII ${ }^{e}$ siècle, toutes les unités d'exploitation, bien qu'indépendantes, se regroupent au sein d'un noyau villageois établi, comme à Yutz, le long d'un axe de circulation héritier du système domanial antique. Dès 711, date obtenue par dendrochronologie sur les bois de fondation du puits, la vie s'organise autour d'un puits collectif. On dénombre au moins six unités d'exploitation sur une surface bâtie de près de 8 ooo $\mathrm{m}^{2}$. La taille des unités varie de $350 \mathrm{~m}^{2}$ pour la plus petite à $1500 \mathrm{~m}^{2}$ pour la plus grande. Chaque unité est composée d'une maison d'habitation et de un ou deux bâtiments à vocation agricole (grange, étable, grenier). Les unités d'exploitation sont séparées les unes des autres par de petites venelles perpendiculaires à la voirie principale. À la toute fin du IX $X^{e}$ siècle (en l'an $900^{3}$ ) et dans la stricte continuité de l'occupation précédemment évoquée ${ }^{4}$, le village adopte une configuration au sein de laquelle les bâtiments s'organisent entre eux pour répondre aux besoins d'un système économique et agraire symptomatique de la polyculture et de pratiques agraires collectives ${ }^{5}$. Dès lors, le village répond à un réel modèle de planification et s'organise selon un schéma très répandu après la guerre de Trente Ans : le villagerue. Celui-ci sera désormais implanté au centre d'un ban évalué à 100 ha, facilitant ainsi l'accès aux champs et réduisant au maximum les déplacements vers le territoire de production. Comme à Yutz, mais avec près de deux siècles d'avance, les champs laniérés vont se juxtaposer les uns aux autres pour former des quartiers

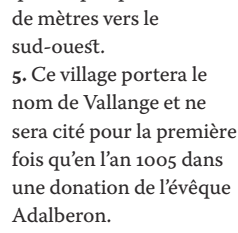


1. Carte de la Lorraine

et localisation des

principaux sites

mentionnés dans l'article.

2. Plan de répartition et

analyse spatiale des

vestiges du bâtiment

principal de Mexy.

3. Évolution de la gestion

d'une unité d'exploitation

à Vitry-sur-Orne entre le

VIII' siècle et le XII siècle.

On transfère les

compétences agricoles

d'une population

organisée en groupes

familiaux auto-

subsistants (VIII-

Ix ${ }^{e}$ siècles) vers une

structure planifiée

associant espace public

et espace privé.

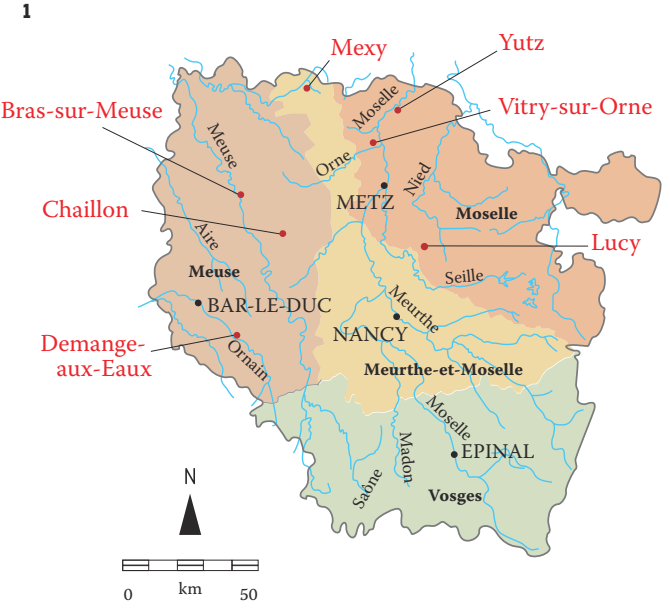

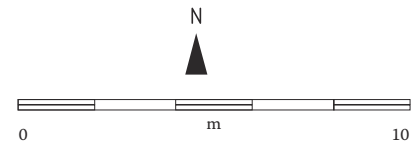

Emplacement supposé d'un bâtiment

Zone d'extension et de répartition à forte concentration de pierres

Zone d'extension maximale et de répartition à faible concentration de pierres

- Blocs probablement en place, posés à plat dans la zone du bâtiment

Do Blocs déplacés

ou à forte probabilité de déplacement

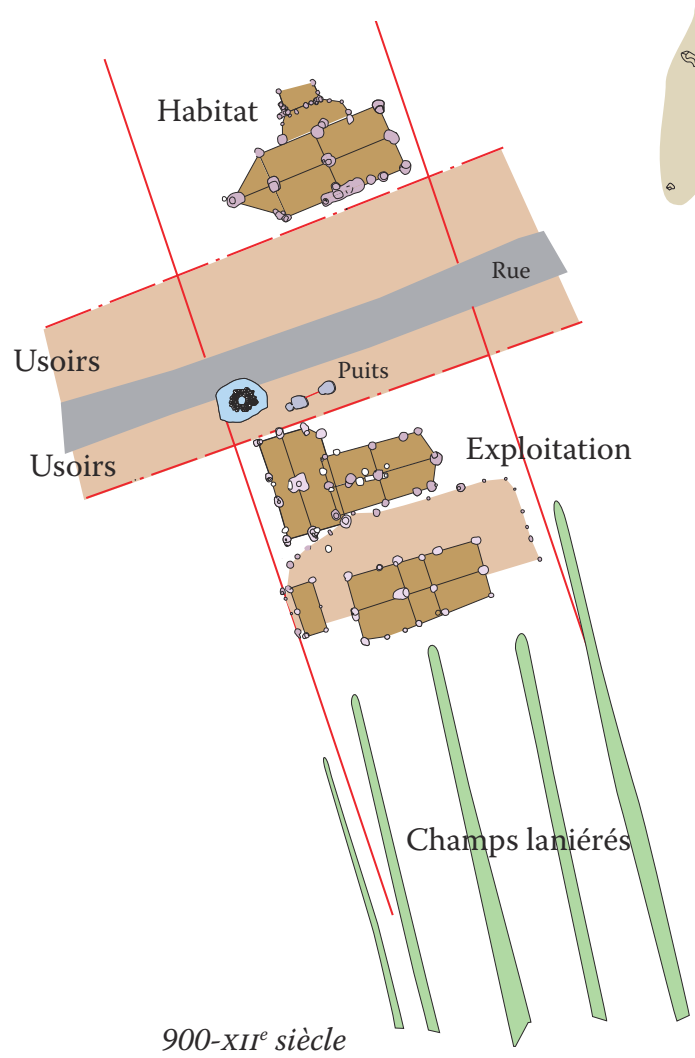

Sens des labours

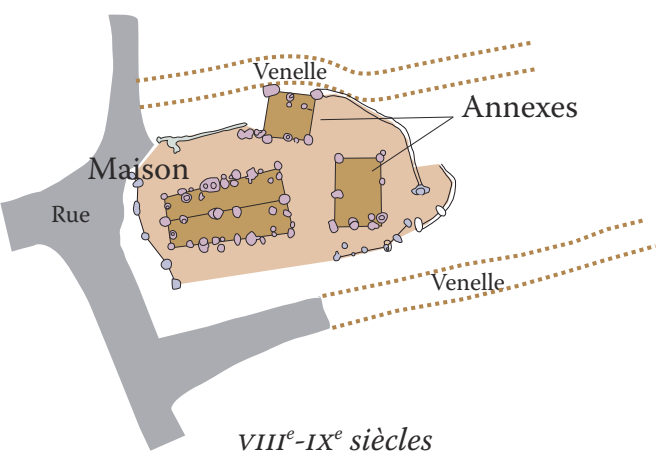

$V I I I^{e}-I X^{e}$ siècles

$\stackrel{\text { m }}{\rightleftharpoons}$


de culture peu ou pas séparés de chemins (Blaising, 2003). Cet enclavement des parcelles constitue la clef de voûte de pratiques agraires collectives puisqu'il nécessitait d'une part une récolte simultanée (et par conséquent un labour et un ensemencement commun) et d'autre part une organisation rigoureuse du travail en récoltant tout d'abord les champs desservis avant de les traverser pour accéder aux parcelles enclavées. L'absence de clôture et de bâtiments en dehors du périmètre villageois défini constitue le second pivot de ce système d'exploitation collectif, en permettant la pratique alternée sur les mêmes terres de cultures et de la vaine pâture. Une fois les récoltes effectuées par chaque propriétaire de parcelles, l'ensemble de ces dernières devient d'usage collectif en étant intégralement dévolu aux troupeaux d'élevage des villageois. C'est la naissance de l'openfield lorrain. Pour faciliter le transfert des populations vers ces nouvelles agglomérations que l'on peut qualifier de « colonisation », les commanditaires assortissaient parfois ces migrations de quelques biens attrayants (Duby, 1962). À Vallange, chaque famille a été dotée d'un puits individuel implanté, préalablement à leur arrivée, en tête de parcelle. Ces nouvelles unités d'exploitation, au nombre de sept, se répartissent le long d'une rue unique de $200 \mathrm{~m}$ qui reprend là encore un axe d'origine antique. Cet axe principal est élargi d'usoirs. Ces espaces, caractéristiques des villages d'agriculteurs, disposent d'un statut particulier pouvant se définir comme une extension du domaine privé. Situés entre la chaussée et les maisons, ces larges espaces restent, même s'ils demeurent communs, d'usage privatif. On y trouve les puits individuels, mais également toutes sortes d'aménagements et de lieux de stockage n'entravant pas de manière irréversible le passage et la circulation (bois, matériels agricoles, fosses à fumier...). Jusqu'au XII ${ }^{\mathrm{e}}$ siècle, chaque unité d'exploitation est divisée en deux cellules distinctes réparties selon le même axe, de part et d'autre de la chaussée et des usuaires. La première cellule comporte un seul bâtiment d'habitation dont les accès s'effectuent côté rue. La seconde cellule, dite d'exploitation, comporte un ou plusieurs bâtiments de taille variable, parfois enclos, dont les ouvertures s'effectuent sur les champs. Les activités domestiques et agricoles sont désormais séparées, avec les hommes d'un côté de la rue et les animaux et les récoltes de l'autre. Tous les bâtiments s'intègrent dans un espace prédéfini, témoin d'une contrainte parcellaire forte qui traduit le côté intangible des limites de chaque unité régie par un cadre juridique parfaitement bien défini, frein considérable aux déplacements. Chaque famille semble ainsi solidement enracinée dans ses parcelles aux bornes desquelles s'arrêtent les servitudes collectives. Cette dernière idée est renforcée par les nombreuses traces de réfection observées sur les bâtiments construits alors selon la technique de poteaux plantés dans le sol. Quelques constructions plus lâches et plus sommaires ont été implantées à l'extrémité est du village (habitat dit «temporaire»). Elles paraissent détachées du noyau villageois et semblent disposer d'un puits collectif, des faits qui laissent penser que ces populations ne jouissent pas des mêmes droits. Il est difficile de qualifier le statut social et juridique de ces habitants de « seconde zone » par l'archéologie ; toutefois, les travaux des champs nécessitant plus de main-d'œuvre à certaines périodes de l'année, il n'est pas exclu qu'il soit régulièrement fait appel à des saisonniers.

À Demange-aux-Eaux, l'habitat groupé se met en place également au $\mathrm{IX}^{\mathrm{e}}$ siècle en s'organisant le long d'un axe de circulation préexistant.

Les bâtiments se développent perpendiculairement à la chaussée selon un rythme d'éloignement de 10 à $30 \mathrm{~m}$. La confrontation du plan de fouille et du cadastre napoléonien est éloquente : chaque bâtiment médiéval se retrouve au sein d'une parcelle dessinée sur le cadastre napoléonien selon un schéma et une orientation tout à fait similaires. Le parcellaire moderne n'est autre que l'héritier d'un parcellaire plus ancien établi au cours du Moyen Âge et perdurant bien au-delà de l'abandon de l'habitat (Gérard, 2012). Comme à Vitry-sur-Orne, il est possible de parler dans ce cas de planification agraire où la structuration de l'habitat est rigoureusement liée aux pratiques agraires collectives. Les parcelles laniérées identifiées à l'arrière des maisons confortent largement cette idée, même si les surfaces étudiées sont nettement moins importantes que dans le cas précédent. Les bâtiments se répartissent en sept unités d'exploitation. Ils sont orientés perpendiculairement à la chaussée, le pignon accolé à la rue ou éloigné de plusieurs mètres. La présence d'usoirs, caractéristiques des villages d'agriculteurs, est ici peu probable. De même, l'organisation et la répartition très strictes observées à Vitry-sur-Orne, où étaient clairement dissociées les zones d'habitat et les zones agricoles (de part et d'autre de la rue), ne sont ici pas évidentes. Chaque unité d'exploitation ne disposant que d'un seul bâtiment, il est judicieux de se poser la question de la cohabitation des hommes et des animaux au sein d'un même édifice. La faible superficie décapée rend la détermination de l'usage domestique, agricole ou artisanal aléatoire, même si dans plusieurs cas la présence de drains d'évacuation permet d'envisager au moins localement une fonction agricole avec stabulation animale. De même, il semblerait que, dans au moins un cas, une activité de pêche soit attestée par la présence récurrente de poids de filets et de lestage qui laissent envisager une spécialisation (saisonnière ou pas) d'au moins une partie de la population vers des activités extra-agricoles [ill. 4]. Le cas de Chaillon

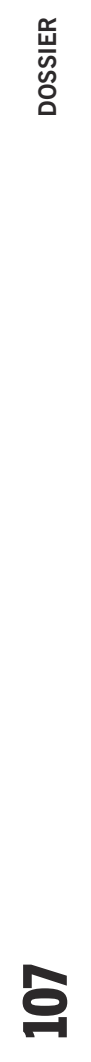




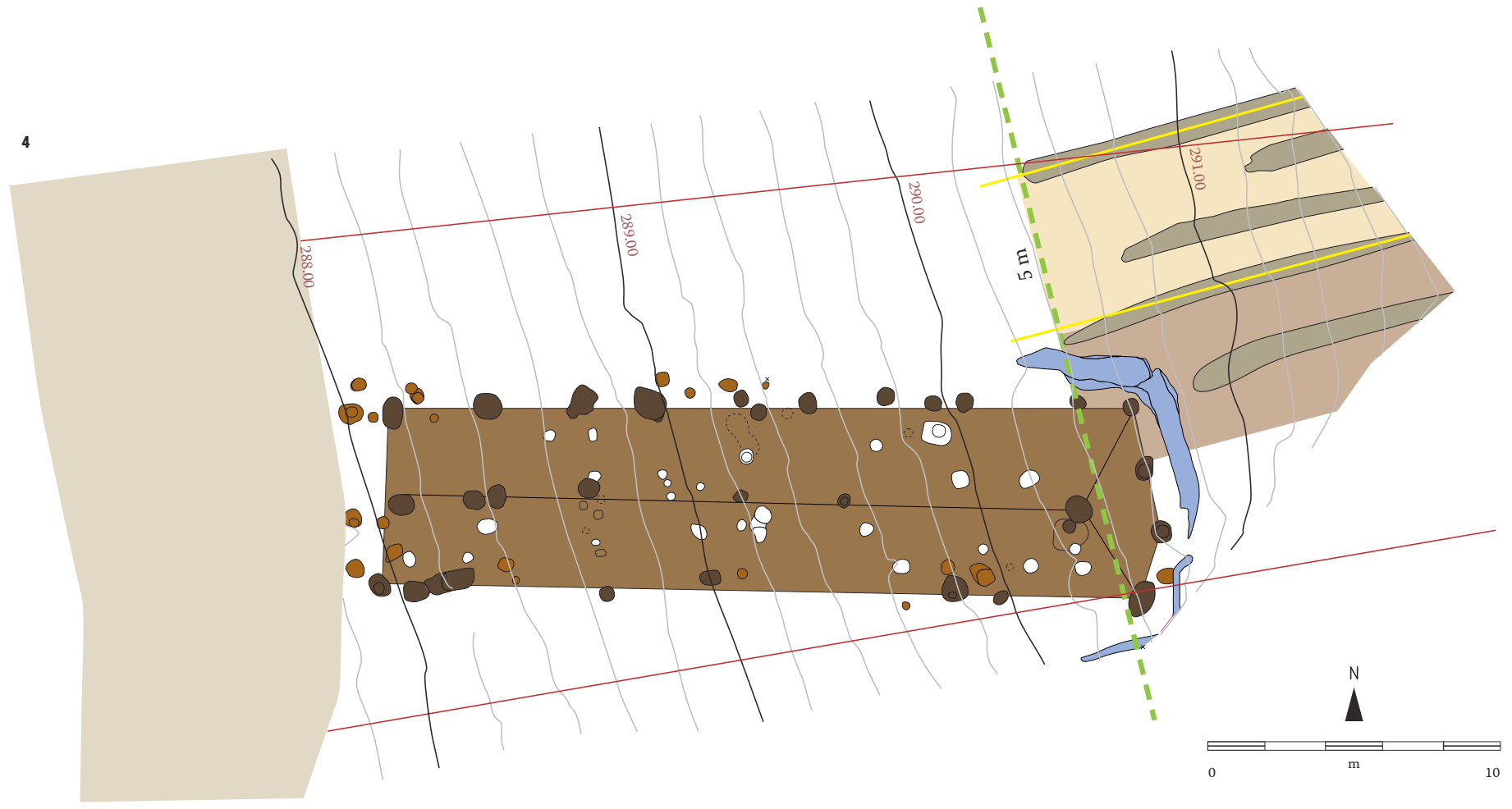

4. Exemple d'une unité d'exploitation des Xe-XIle siècles à Demange-auxEaux. À l'arrière de cette longue maison se trouvent les parcelles Ianiérées qui trahissent une évidente activité agricole axée sur la polyculture et le collectivisme.Les nombreux poids de filets de pêche et de lestage

\section{témoignent cependant} d'une seconde activité complémentaire

pratiquée par les

membres de cette unité d'exploitation.

5. Les principaux types de

bâtiments à poteaux plantés dans le sol.

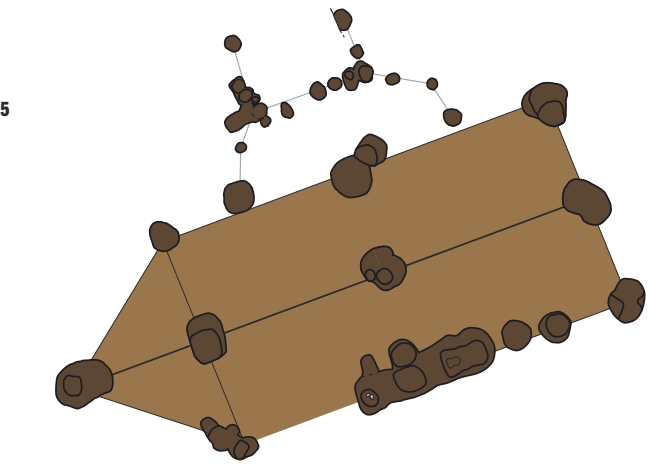

Maison à poteaux porteurs de la panne faîtière $\left(\mathrm{X}^{\mathrm{e}}\right.$-XII ${ }^{\mathrm{e}}$ siècles) - Vitry-sur-Orne

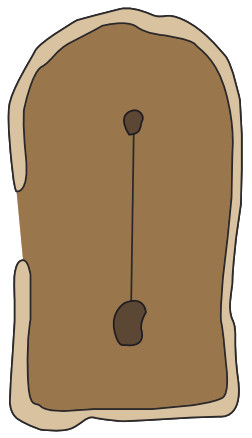

Maison à poteaux porteurs de la panne faîtière et murs en terre fondés $\left(\mathrm{VIII}^{\mathrm{e}}-\mathrm{X}^{\mathrm{e}}\right.$ siècles $)$ - Yutz

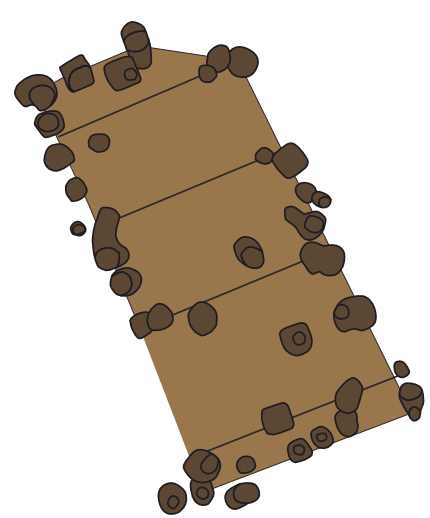

Maison à entraits $\left(\mathrm{X}^{\mathrm{e}}-\mathrm{XII}^{\mathrm{e}}\right.$ siècles $)$ Vitry-sur-Orne

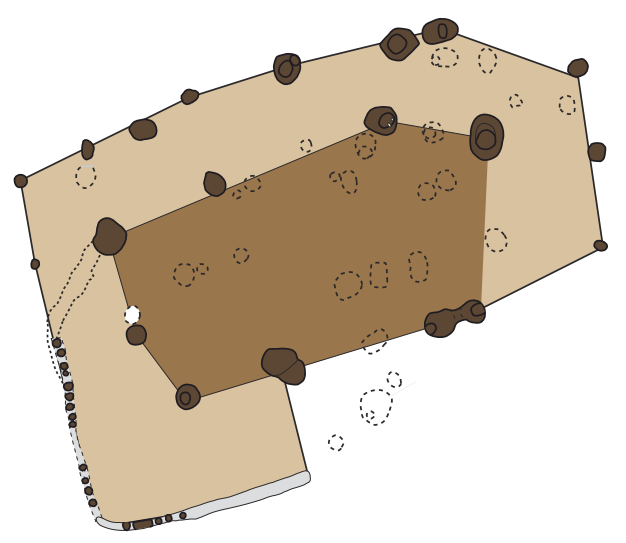

Maison à cadre interne ( $\mathrm{X}^{\mathrm{e}}-\mathrm{XII}^{\mathrm{e}}$ siècles $)$ Vitry-sur-Orne 
est différent. Si l'habitat groupé est également attesté autour du $\mathrm{X}^{\mathrm{e}}$ siècle, il est ici prématuré de parler de planification ou de pratique agraire collective. Le site s'organise le long de l'actuelle route, déjà en service au cours du Moyen Âge. Ce sont sept bâtiments à poteaux plantés dans le sol qui ont été mis au jour dans l'emprise de la fouille. Ils s'organisent tantôt perpendiculairement, tantôt parallèlement à la chaussée selon un rythme régulier de 2, 4, 6 et $10 \mathrm{~m}$ de distance entre eux. Les façades et pignons ne sont pas strictement alignés malgré leur parfaite organisation orthogonale. La distance qui sépare les bâtiments de la chaussée varie de $1 \mathrm{~m}$ à $7 \mathrm{~m}$. Comme dans le cas de Demange-aux-Eaux, les usoirs, caractéristiques, rappelons-le, des villages d'agriculteurs, sont inexistants à Chaillon, où les activités agricoles demeurent une pratique récurrente, attestée par les études carpologiques et archéozoologiques, mais également par la présence ponctuelle dans certains bâtiments de drains d'évacuation de purin. Les deux fours de potier relégués à l'arrière des parcelles bâties orientent toutefois la population vers une activité principale consacrée à l'artisanat et plus spécifiquement à la production de céramique. Ce statut spécifique sera conforté par le développement architectural et spatial de ces maisons dès le XIII ${ }^{\mathrm{e}}$ siècle...

Jusqu'au XII ${ }^{\mathrm{e}}$ siècle, de façon quasi générale, les bâtiments ruraux étaient construits en matériaux périssables et disposaient de fondations constituées uniquement de poteaux plantés dans le sol, poteaux généralement en chêne (bois dur, résistant, élastique et durable) qui serviront de base à l'élévation des édifices. Si cette technique assure leur résistance aux efforts latéraux (particulièrement ceux dus au vent), elle présente de nombreuses contraintes techniques liées à l'entretien des poteaux. Les études réalisées à partir de modèles récents montrent que les parties souterraines (humides mais à l'abri de l'air) et aériennes (au sec et ventilées) des poteaux ne posaient pas de véritables problèmes de conservation. Seule celle située au ras du sol, sur environ $10 \mathrm{~cm}$, était sujette, par la présence constante d'humidité et d'air, au développement de moisissures pouvant altérer les fibres du bois jusqu'à les détruire. En l'absence de réparations, les bâtiments ne pouvaient résister plus de 30 à 50 années. L'analyse archéologique de chacun de ces vestiges montre que les poteaux de ces bâtiments ont très majoritairement fait l'objet tantôt de remplacement, tantôt de renforcement rendant ainsi la lecture du sol difficile et transformant une simple paroi de bâtiment en un chapelet parfois continu de fosses d'implantation de poteaux pas toujours contemporains les uns des autres. Ce constat traduit également les limites intangibles des parcelles dans lesquelles s'inscrivent ces édifices, chaque villageois respectant le maintien, parfois pendant plusieurs générations, des maisons au même endroit. L'ossature architecturale de ces bâtiments ne diffère que très peu d'un site à l'autre. Si l'on exclut l'édifice de son contexte général et de son implantation au sein du village et que l'on s'appuie uniquement sur des critères morphologiques, il est souvent très difficile d'en déterminer l'usage et la fonction. Les bâtiments se répartissent en deux grands ensembles de développement architectural [ill. 5]. Le plus fréquent est incontestablement celui à support direct de la panne faîtière que l'on retrouve de manière exclusive à Yutz, mais également fréquemment à Vitry-sur-Orne et Demange-aux-Eaux et de manière plus sporadique à Chaillon. Les poteaux de parois supportent une sablière haute reliée à la panne faîtière par des chevrons. À Yutz, ces bâtiments présentent la particularité de disposer parfois d'un pignon en abside constitué de murs en terre ancrés dans des fondations peu profondes. Ces murs sont constitués d'un épais entrelacs de branchages maintenus par une ossature de piquets, le tout étant enduit généralement de torchis. Cette technique de clayonnage permet de mettre en œuvre des tracés sinueux sans négliger une cohésion mécanique efficace de l'édifice (Blaising, 2004). Le second type de bâtiment présente une architecture constituée d'entraits sur lesquels reposent parfois des poinçons supportant la panne faîtière. Ces bâtiments sont généralement plus rares, mais ils semblent majoritaires sur le site de Chaillon. Ils offrent l'avantage de disposer d'un espace au sol libéré de toutes contraintes techniques liées à la présence de poteaux verticaux d'une seule pièce du sol au faîte du toit dans l'axe de l'édifice. Certains bâtiments associent les deux techniques en intégrant entraits et supports directs de la panne faîtière. Cette mixité, que l'on retrouve de façon récurrente sur la quasi-totalité des sites, peut résulter de la mise en œuvre de renforts ponctuels au cours de l'entretien des édifices. Enfin, un dernier type de bâtiment, nettement plus rare, présente une architecture à cadre interne. Un exemplaire a été mis au jour dans la partie agricole d'une unité d'exploitation du village de Vitry-sur-Orne (Vallange). La couverture des bâtiments semble exclusivement constituée de matériaux périssables sans que l'on puisse avec certitude mentionner leur nature (paille de seigle, bardeaux de bois...). L'absence récurrente de niveaux de sol, même dans le cas de bâtiments fossilisés par des installations postérieures, permet de s'interroger sur la nature exacte de certains édifices et sur l'hypothèse de bâtiments disposant parfois d'un plancher surélevé, solution efficace contre les remontées d'humidité.

Concernant la morphologie des édifices, on note une importante disparité que ce soit d'un village à l'autre ou au sein d'une même agglomération. Si une large majorité des bâtiments présentent un plan rectangulaire (parfois trapézoïdal) et que leur largeur est 


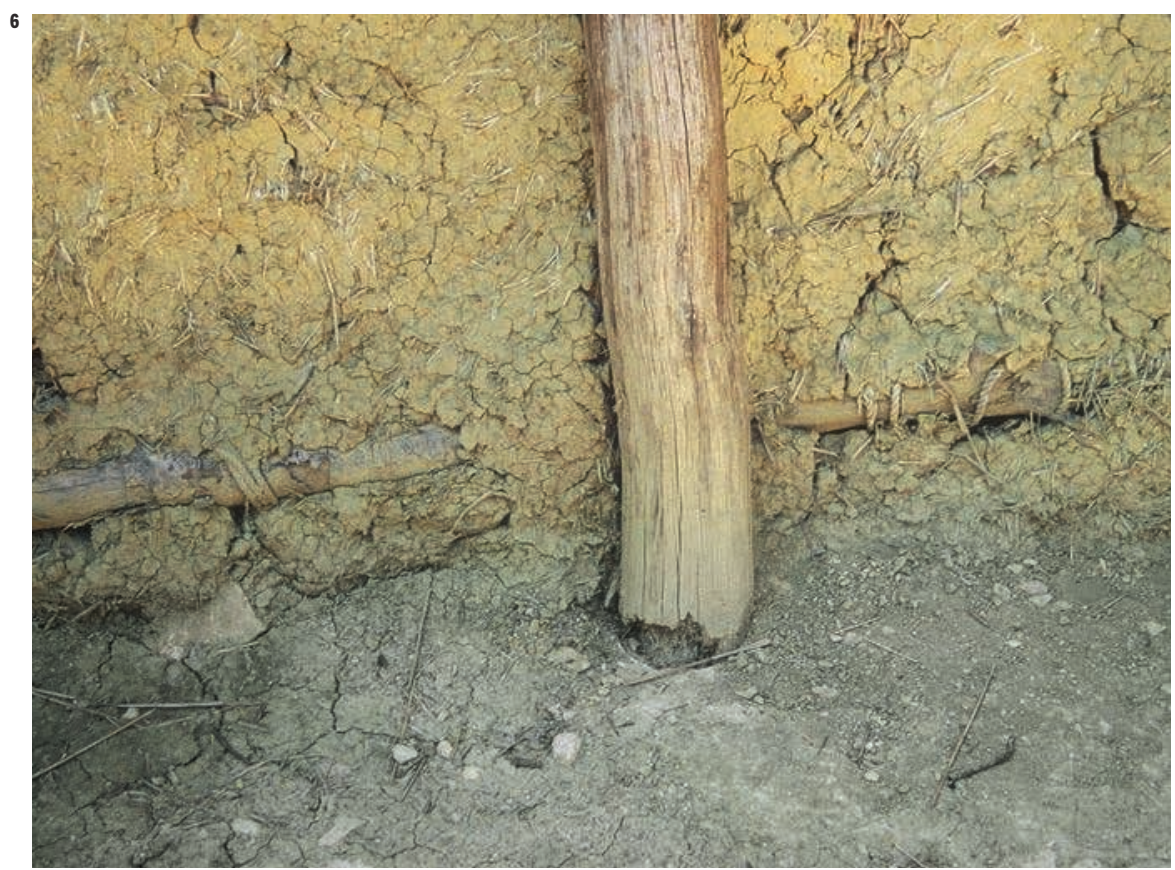

6. En haut, exemple de

poteau planté dans le sol

(archéodrome de Beaune)

et d'altération du bois

à la base du poteau en

contact permanent avec

l'air et de l'humidité ;

en bas (sud du Laos,

plateau des Bolovens,

minorités ethniques

des Laven), les poteaux

reposent sur des dés

de pierre empêchant

toute altération du bois.

7. Les différentes

techniques de solins:

A: poteau reposant sur

sablière basse elle-même

supportée par un

alignement sommaire de

blocs disposés sur le sol,

B: poteau reposant sur

une sablière basse

disposée sur un

soubassement de mur

continu légèrement

fondé,

C: poteau reposant sur un

dé de pierre disposé sur

le sol avec sablière basse

et solins discontinus,

D: poteau reposant sur

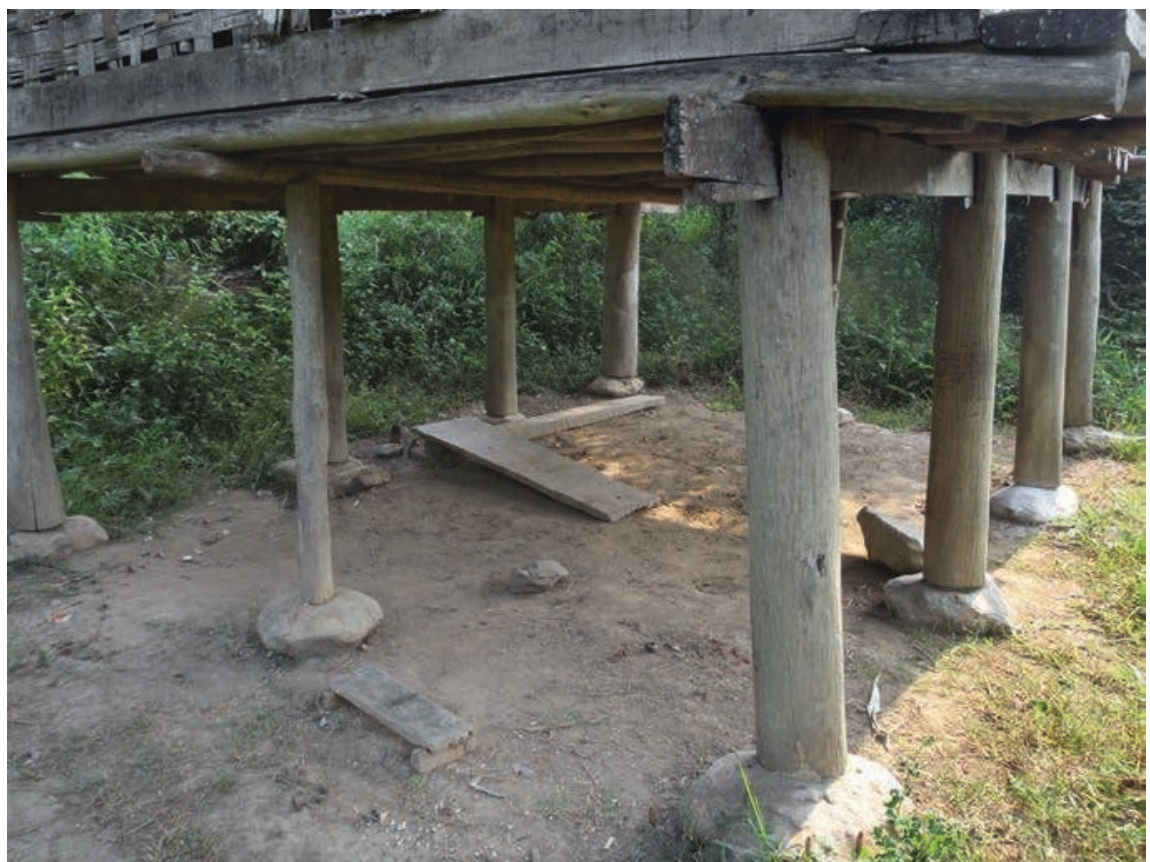

un dé de pierre disposé

sur le sol sans sablière

basse ni solin.

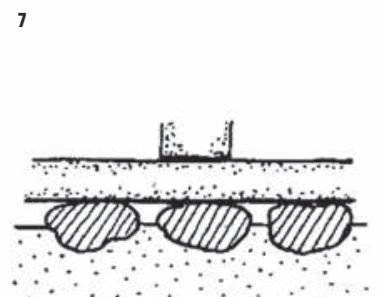

A

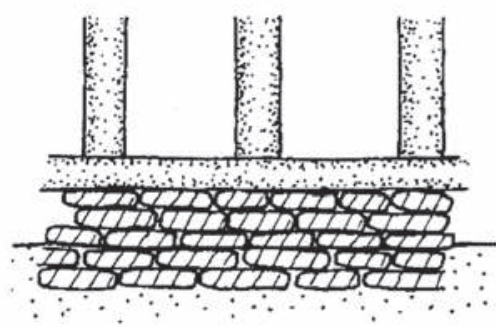

B

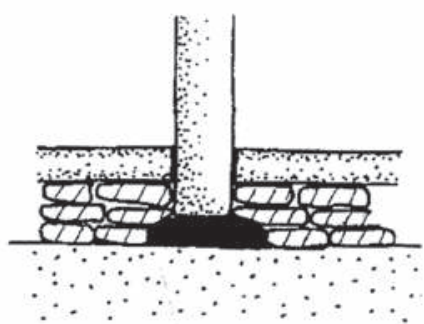

C

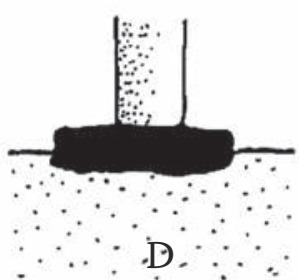


toujours comprise entre $5 \mathrm{~m}$ et $8 \mathrm{~m}$ (pour des raisons techniques liées aux portées maximales des poutres horizontales), leur longueur varie fortement. Certains édifices sont relativement courts avec des longueurs comprises entre $5 \mathrm{~m}$ et $10 \mathrm{~m}$ et d'autres excessivement longs avec des cotes parfois supérieures à $20 \mathrm{~m}$ comme à Demange-aux-Eaux. Ce constat explique la forte disparité des indices d'allongement et de la superficie au sol des bâtiments, même si la plupart d'entres eux accusent des surfaces comprises entre 50 et $70 \mathrm{~m}^{2}$.

\section{Un nouveau type d'architecture à partir du XIII ${ }^{\mathrm{e}}$ siècle : la maison-bloc en pan de bois sur solins de pierres}

Dès les XII ${ }^{\mathrm{e}}$-XIII ${ }^{\mathrm{e}}$ siècles, les communautés villageoises s'installent dans la pérennité, condition sine qua non au bon fonctionnement du système collectif de l'openfield lorrain. Cette stabilité va s'accompagner de quelques modifications architecturales liées à une meilleure maîtrise des techniques de construction [ill. 6]. Pour pallier les travaux fastidieux et répétitifs d'entretien des maisons, qui venaient s'additionner à ceux des champs, les charpentiers alors cantonnés aux milieux aristocratiques et religieux vont progressivement investir le monde rural. Dès les $\mathrm{XII}^{\mathrm{e}}$-XIII ${ }^{\mathrm{e}}$ siècles, ils vont mettre en œuvre de nouvelles techniques qui vont profondément modifier la vie des paysans (Gérard, 2009).

Pour éviter la remontée par capillarité de l'eau le long des poteaux plantés dans le sol, ces derniers vont désormais reposer sur des dés de pierre ou sur des sablières basses assises elles-mêmes sur des solins de pierres [ill. 7]. À Yutz, cette technique ne sera pas immédiatement maîtrisée, comme l'attestent plusieurs cas de sablières en bois posées dans des tranchées (Blaising, 2007). La stabilité de l'ensemble sera assurée par des liens de contreventement qui rattacheront alors les poteaux verticaux aux poutres horizontales. Le pan de bois est né, conférant aux bâtiments une relative souplesse largement appropriée aux sols argileux lorrains. Dès lors, et jusqu'aux $\mathrm{Xv}^{\mathrm{e}}$ $\mathrm{XVI}^{\mathrm{e}}$ siècles, cette technique va être mise en œuvre sur l'ensemble des sites énoncés à l'exception de celui de Demange-aux-Eaux, qui sera définitivement abandonné au XIII ${ }^{\mathrm{e}}$ siècle pour laisser place intégralement à l'extension des zones cultivées (probable déplacement de l'habitat). Ailleurs, cette prouesse technique va permettre de construire de vastes maisons moins difficile à entretenir. Si le strict cadre juridique régit toujours le parcellaire de la communauté villageoise, cette innovation architecturale s'accompagne d'un profond bouleversement dans l'organisation des cellules familiales. Ce nouveau dispositif traduira très vite une véritable identité sociologique de la maison qui devient un signe de reconnaissance sociale et professionnelle de l'individu...
La maison comme identité sociologique et professionnelle

À Vitry-sur-Orne (Vallange), les activités agricoles et domestiques sont regroupées sous un même toit au sein d'une seule maison, d'un côté de la rue. Le nombre de bâtiments se voit réduit, libérant ainsi de l'espace voué à l'agrandissement des parcelles cultivées. On parle alors de la maison-bloc où l'homme partage ses murs avec les animaux et les récoltes, selon une organisation bien définie de l'espace. De rares bâtiments à poteaux plantés dans le sol (interprétés comme des greniers) persistent en périphérie de certaines maisons-blocs. À quelques pas de là, toujours sur la commune de Vitry-sur-Orne, à l'emplacement du hameau de Huppigny au pied de la butte de Justemont, le même phénomène se produit, mais toutes les maisons sont pourvues de caves et aucun usoir ne sépare la bâtisse de la chaussée contre laquelle elle est accolée. Cette organisation est caractéristique des maisons de vignerons, activité confirmée par les études carpologiques réalisées sur le site (Lansival, 2005). On notera donc à Vitry-sur-Orne une volonté d'implanter les communautés villageoises sur le territoire en fonction de leur activité professionnelle, les agriculteurs au centre du ban et les vignerons au pied des côtes. Cet exemple est également symptomatique de l'adéquation entre la maison et l'activité des populations. À Chaillon, on observe le même phénomène de rétrécissement de l'espace bâti et d'adaptation de la maison aux activités professionnelles des villageois. Les bâtiments à poteaux plantés dans le sol sont remplacés par de grandes maisons en pan de bois sur solins de pierres se présentant sous la forme d'une enfilade de pièces toutes ou presque pourvues de cheminées, laissant ainsi peu ou pas de place aux animaux, au dépôt du matériel agricole et au stockage des récoltes. Chaque bâtiment paraît rassembler sous un même toit plusieurs familles, vivant chacune dans une vaste pièce pourvue d'un foyer et ouvrant directement sur les venelles perpendiculaires à la rue principale. Ce dispositif n'est pas sans rappeler le statut d'artisan-potier envisagé dès le $\mathrm{x}^{\mathrm{e}}$ siècle pour la population de ce village. Le cas de Lucy est plus atypique, mais il n'en demeure pas moins un nouvel exemple d'adaptation de la maison rurale aux activités professionnelles de ses habitants, tant dans son implantation géographique que dans sa configuration interne. Créé ex nihilo au XIII ${ }^{\mathrm{e}}$ siècle, il prend immédiatement la configuration d'une maison à vocation agricole dans laquelle cohabitent hommes et animaux. Les études archéozoologiques ont permis de préciser l'activité agricole en l'orientant vers l'élevage de caprinés, ce qui explique le caractère totalement isolé, hors de toute structure villageoise, de cette imposante bâtisse (bergerie). 


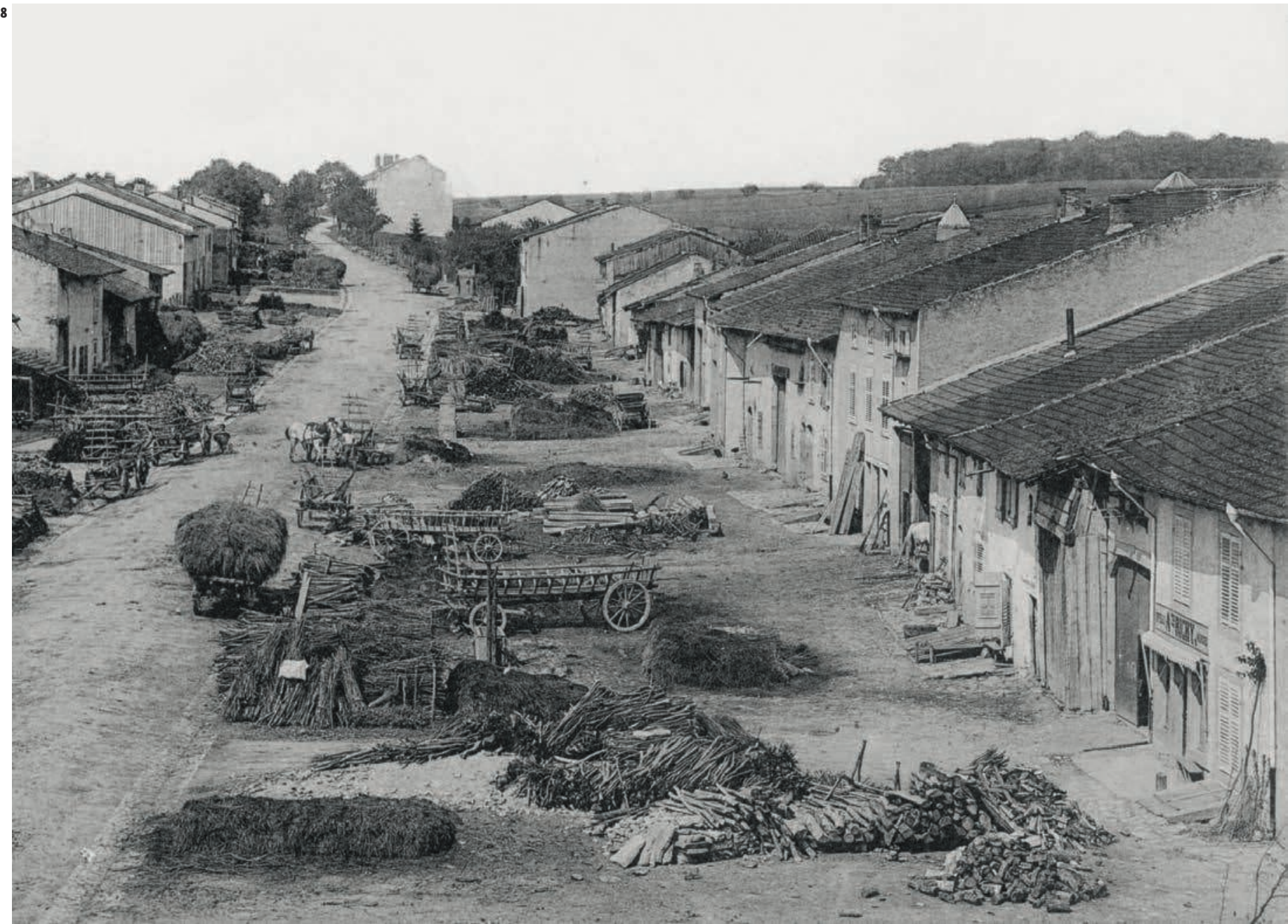

8. Carte postale ancienne

d'un village lorrain au

début du xxe siècle;

on perçoit nettement

l'identité du village-rue

avec sa rue principale,

ses usoirs où sont

entreposés les machines

agricoles, le bois et

le fumier ainsi que ses

maisons de laboureur

en enfilade. 
Et après...

De nombreux villages ne résistent pas aux crises économiques et démographiques du $\mathrm{XV}^{\mathrm{e}}$ siècle et sont tout simplement abandonnés, comme dans le cas de Vallange, dont le ban sera rattaché à celui de Vitry-sur-Orne. D'autres villages seront rayés de la carte au XVII ${ }^{\mathrm{e}}$ siècle, à la suite de l'effroyable guerre de Trente Ans qui anéantit les populations obligées de fuir une nouvelle fois leurs terres. Ce sont spécifiquement ces villages qui, de par leur emplacement à l'écart des agglomérations actuelles et donc à l'endroit même des projets d'aménagement du territoire, font l'objet du plus grand nombre d'investigations archéologiques. Les communautés les plus résistantes face aux fléaux économiques et politiques de ces périodes troubles continueront à évoluer jusqu'à nos jours. Elles s'adapteront à de nouvelles volontés politiques, à de nouvelles orientations économiques et à de nouvelles techniques agricoles. Le village restera un outil de production, mais les maisons en pan de bois seront progressivement remplacées par les maisons en pierre (à l'exception de quelques régions comme l'Argonne ou l'est mosellan dans lesquelles le bois restera encore utilisé longtemps). Ces innovations permettront un nouveau resserrement de l'habitat et l'apparition de la mitoyenneté jusque-là impossible par souci de propagation des incendies. L'archéologie du soussol laisse place à l'archéologie du bâti, à la géographie et à l'iconographie... [ill. 8] Les structures parcellaires et urbaines du village ne disparaissent que depuis peu avec la mise en œuvre des remembrements et l'apparition aussi spontanée que rapide d'habitations neuves et de lotissements résidentiels en dehors des noyaux anciens des villages. Dans le même temps, le nombre de fermes diminue constamment pour ne former plus qu'une seule grande exploitation, implantée le plus souvent en bordure du village selon un modèle qui rappelle celui des domaines agricoles antiques [ill. 12]. Si la maison garde une identité sociologique, elle voit sa qualité d'outil disparaître au même titre que le village qui, au terme de plus de 1 ooo ans de mise en œuvre, risque de perdre bientôt sa ruralité...

\section{Références bibliographiques}

BLAISING J.-M., 2003, « Parcellaire laniéré, billons et crêtes de labours, les structures agraires en Lorraine du XII ${ }^{\mathrm{e}}$ s. à hier ", in Sols et structures agraires, Actes de la table ronde de Sarrebourg, 10-11 octobre 2003, Sarrebourg, Inrap, p. 45-52.

BLAISING J.-M., 2004, « L'apport des sources iconographiques à l'archéologie de la construction en terre et bois en pays thionvillois ", in NÈGRE V. (DIR.), Terre crue, terre cuite. Recueil d'écrits sur la construction, Paris, Ibis Press, p. 41-52.

Blaising J.-M., 2007, « Haute-Yutz, le temps d'un village », Les Cahiers lorrains, 3/4, p. 13-33.

DuBy G., 1962, L'Économie rurale et la vie des campagnes dans l'Occident médiéval (France, Angleterre, Empire, $I \mathrm{X}^{e}$-XV $\mathrm{V}^{e}$ siècles). Essai de synthèse et perspectives de recherches, Paris, Aubier, 2 vol., 823 p.

Gérard C., Peltre J., 1979, Les Villages lorrains, Nancy, Université de Nancy II, 105 p [épuisé].

GÉRARD C., 1982, « Les caractéristiques du village lorrain ", in CABOURdin G, LANHER J. (DIR.), Villages et maisons de Lorraine, Actes du colloque de Nancy, 22-24 octobre 1981, Nancy/Metz, Presses universitaires de Nancy/éd. Serpenoise, p. 9-20.

GÉRARD C., 1990, La Maison rurale en Lorraine [contribution à un inventaire régional], Nonette, éd. Créer, 151 p.

GÉrARd F., Blaising J.-M., 2007, « Techniques de construction et structure du village en basse vallée de Moselle du VIII ${ }^{\mathrm{e}}$ siècle au $\mathrm{XX}^{\mathrm{e}}$ siècle, in Trochet J.-R. (DIR.), Les Maisons paysannes en Europe occidentale de la fin du Moyen Âge au $X X^{e}$ siècle, Actes du colloque Paris Sorbonne, 14-16 septembre 2006, Paris, Pups, p. 165-179.

GERARD F., 2009, « Un exemple d'architecture en pan de bois sur solin de pierres à Vallange $\left(\mathrm{XIII}^{\mathrm{e}}-\mathrm{XV}^{\mathrm{e}}\right)$ », in La Construction des maisons au XV siècle en Alsace et dans la région du Rhin supérieur, Actes du colloque de Wissembourg, 10-14 octobre 2007, Marburg, Jonas Verlag, p. 183-197.

GÉRARD F., 2012, « La structuration du village pour une économie agraire planifiée. L'apparition du parcellaire laniéré en Lorraine à la fin du $\mathrm{IX}^{\mathrm{e}}$ siècle, l'exemple des sites de Vitry-sur-Orne (Vallange) et de Demange-aux-Eaux », Archéopages, 34, p. 38-47.

LANSIVAL R., 2005, « Les bâtiments ruraux du bas Moyen Âge de Vitry-sur-Orne (vr. 52) », Les Cahiers lorrains, 4, p. 261-273.

Peltre J., 1981, « Les espaces collectifs villageois, l'exemple des usoirs lorrains », in CABOURDIN G, LANHER J. (DIR.), Villages et maisons de Lorraine, Actes du colloque de Nancy, 22-24. octobre 1981, Nancy/Metz, Presses universitaires de Nancy/éd. Serpenoise, p. 39-46.

Peltre J., 1989, « Bans et villages : une longue histoire, un avenir en question », in R. TAVENEAUX (DIR.), Encyclopédie illustrée de la Lorraine : la vie traditionnelle, Metz/Nancy, éd. Serpenoise p. 151-158.

ZimmermanN W.-H., 1998, Probleme der Küstenforschung im Südlichen Nordseegebie, Oldenburg, Isensee Verlag, vol. 25. 


\section{Une maison de laboureur à Vitry-sur- Orne}

Cette maison-bloc intègre une parcelle de $30 \mathrm{~m}$ de large. Il s'agit d'une maison unifaîtière à quatre travées (deux travées «logis » correspondant à deux cinquièmes du bâtiment et deux travées "exploitation " correspondant à trois cinquièmes du bâtiment). Flle occupe une superficie totale d'environ $250 \mathrm{~m}^{2}$ et présente un indice d'allongement de 1,78 (longueur : $25 \mathrm{~m}$; largeur maximale : $14 \mathrm{~m}$ ) [ill.1].

Le logis est orienté perpendiculairement au faîtage. Il occupe les deux cinquièmes est du bâtiment (travées 1 et 2 ), soit une superficie totale de $100 \mathrm{~m}^{2}$. Il est composé de quatre pièces ou ensembles, distribués par un couloir traversant le logis de part en part, depuis l'usoir jusqu'au jardin. Il est long de $10 \mathrm{~m}$ et large de $2,50 \mathrm{~m}$ environ. Un accès de $0,80 \mathrm{~m}$ de large, délimité au sol par deux imposants blocs calcaires, a été identifié à l'arrière de la bâtisse. La cuisine se situe dans l'angle nord-est de la maison, côté rue. Longue de 5,50 m et large de $2,50 \mathrm{~m}$, elle occupe une superficie au sol de $13,75 \mathrm{~m}^{2}$. Une cheminée a été identifiée sur le pignon est de la maison, dans l'angle sud-est de la cuisine. Dans le prolongement de la cuisine se trouve une pièce de type "chambre». Flle mesure ", $5 \mathrm{~m}^{2}$ (longueur : $3 \mathrm{~m}$; largeur : $2,50 \mathrm{~m}$ ). La partie ouest du logis, de l'autre côté du couloir de distribution, est occupée par un grand ensemble de $25 \mathrm{~m}$. Les observations archéologiques ne permettent pas de dissocier cet ensemble en plusieurs entités. Seule la chambre à four qui occupe la partie nord-ouest du logis ( $5 \mathrm{~m}^{2}$ environ) a été reconnue. Aucune trace archéologique de cloisons ou de mur de séparation ne permet de la dissocier d'une éventuelle chambre installée dans son prolongement sud. L'exploitation occupe les trois cinquièmes ouest de l'édifice soit une superficie totale d'environ $150 \mathrm{~m}^{2}$. La lecture archéologique de cet ensemble rend difficile toute interprétation détaillée des espaces et de leurs fonctions. Toutefois, plusieurs éléments se dégagent et permettent de localiser une stalle, l'étable et éventuellement la porcherie.L'étable est située transversalement au faîtage de la maison, le long du mur de séparation du logis et de l'exploitation. Flle est large de $\mathrm{Z} \mathrm{m}$ et longue de $8 \mathrm{~m}\left(16 \mathrm{~m}^{2}\right)$. Une stalle de $4 \mathrm{~m}^{2}$ est délimitée par un mur de refend de $2 \mathrm{~m}$ de long, perpendiculaire au mur gouttereau de façade. Un drain d'évacuation s'échappe de l'étable en direction des jardins, à l'arrière de la maison. Une petite annexe de type porcherie (?), contemporaine de la construction, est accolée au mur de façade de la maison, au milieu de la partie exploitation. Flle dispose d'une ouverture sur la rue de $1 \mathrm{~m}$ de large. Longue de $2,50 \mathrm{~m}$ et large de $2,50 \mathrm{~m}$, elle dispose d'une superficie au sol de $6,25 \mathrm{~m}^{2}$. 


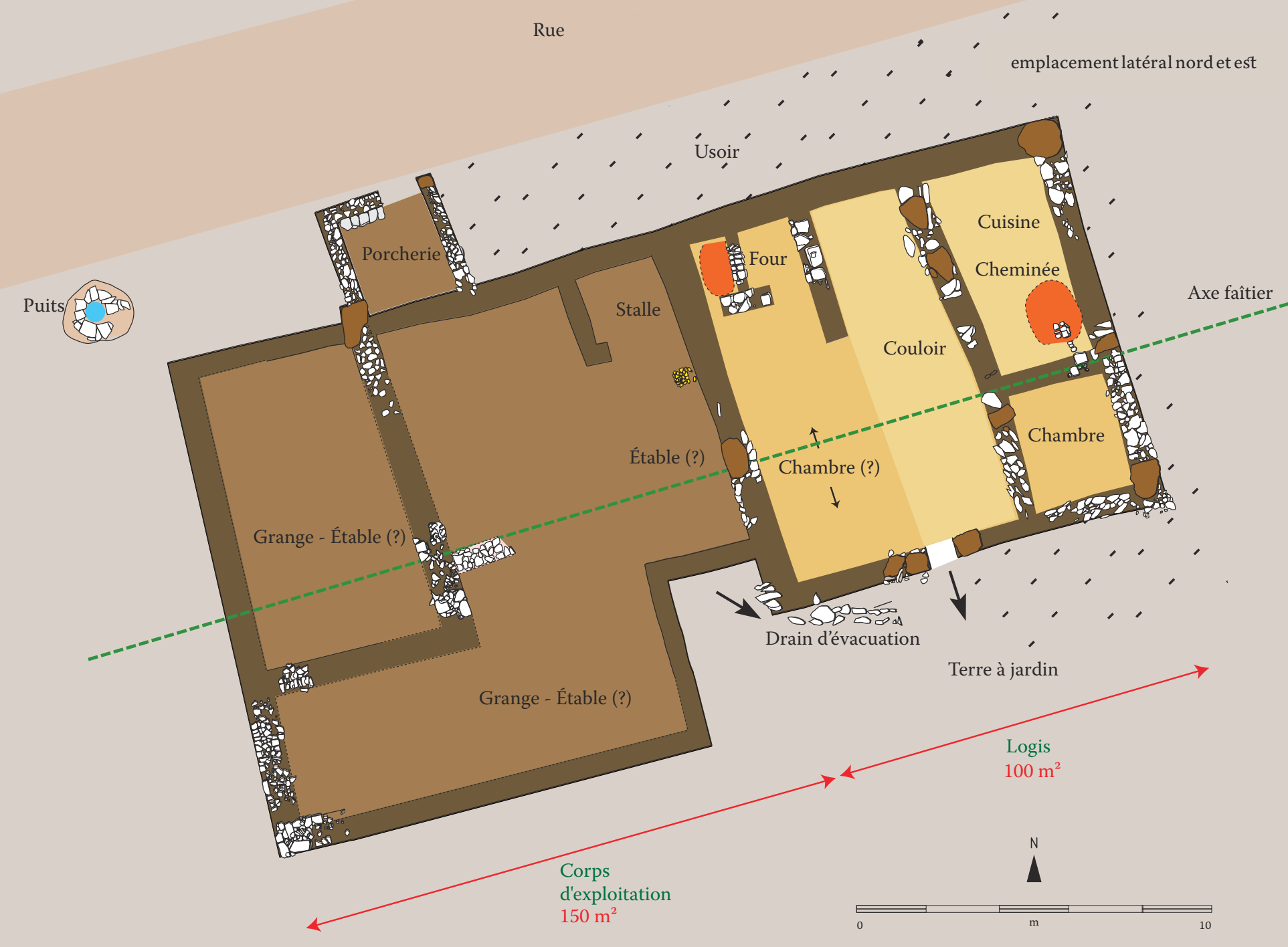

Le mode de construction des murs, ainsi que les états successifs d'occupation et de réaménagement témoignent du caractère probablement détrempé et boueux des lieux. \#n effet, si l'ossature générale de cette annexe demeure en pan de bois (en témoignent les gros dés de pierre installés dans les angles), le remplissage des murs semble être composé de pierres. Le sol de la pièce, en terre battue sur hérisson de pierres, a quant à lui subi maintes réfections. Il est impossible de qualifier et de définir les autres espaces de l'exploitation. Une pièce de $36 \mathrm{~m}^{2}$ (4,50 $\mathrm{m}$ de large en moyenne et $8 \mathrm{~m}$ de long) occupe l'angle nord-ouest de la bâtisse perpendiculairement au faîtage tandis qu'un vaste espace ouvert d'environ $80 \mathrm{~m}^{2}$ la sépare de l'étable $\left(40 \mathrm{~m}^{2}\right.$ perpendiculaire au faîtage et $40 \mathrm{~m}^{2}$ parallèle à celui-ci, à l'arrière de la maison). Cet espace couvre ainsi la maison depuis le mur de façade jusqu'au mur gouttereau arrière. Le faîtage, disposé dans l'axe de l'étable et donc aux deux tiers du logis, favorise l'hypothèse d'un toit à deux pans asymétriques et allongés l'un vers la rue et l'autre vers les jardins. La toiture semble garnie de tuiles canal à crochet découvertes en grand nombre dans les niveaux de démolition de la bâtisse. Ce type de couverture paraît plus approprié que le chaume en raison de la proximité immédiate d'une autre bâtisse du côté est et des risques d'incendie qui en découlent. Il est important de noter l'absence de couloir de distribution entre le logis et l'exploitation ainsi que l'absence de porte charretière et de caves que l'on retrouvera de façon récurrente sur les maisons de vignerons du hameau voisin . 
Ces deux édifices présentent de nombreuses similitudes architecturales, mais se différencient par leur dimension et leur distribution [ill.1]. Les deux bâtiments sont orientés de la même manière, perpendiculaires à la chaussée, et respectent une inclinaison de $65^{\circ}$ nord-est. Le bâtiment 1, long de $36 \mathrm{~m}$, dispose d'une largeur comprise entre 8 $m$ (pour les trois quarts de l'édifice) et $12 \mathrm{~m}$ (pour le quart restant), soit une superficie importante de $328 \mathrm{~m}^{2}$. Le bâtiment 2 est nettement plus petit avec une longueur de $17 \mathrm{~m}$ et une largeur constante de $10 \mathrm{~m}$, soit une superficie de $170 \mathrm{~m}^{2}$. Les deux édifices présentent la même configuration principale avec une enfilade de pièces de taille importante (quatre pièces de $56 \mathrm{~m}^{2}$ à $72 \mathrm{~m}^{2}$ pour le bâtiment 1 et deux pièces de $35 \mathrm{~m}^{2}$ à 46 $\mathrm{m}^{2}$ pour le bâtiment $\mathrm{Z}$ ). Le second bâtiment présente la particularité de bénéficier d'espaces supplémentaires matérialisés par une tour d'angle, un vestibule et une annexe latérale. La présence d'une tour d'angle laisse supposer l'existence d'un étage. Les deux bâtiments sont intégralement construits selon la méthode du pan de bois sur solins de pierres. Les murs, non maçonnés, ne sont généralement pas fondés, à l'exception de quelques portions sur le bâtiment 2 , où un soin particulier a été apporté au montage des solins et où on peut observer une réelle volonté de stabiliser l'édifice en fondant certains murs aux emplacements d'anciennes excavations. Ia largeur moyenne des solins est de $0,70 \mathrm{~m}$ à 0,80 $\mathrm{m}$. Ils ne sont généralement constitués que d'un seul rang de pierres de $0,20 \mathrm{~m}$ de hauteur. Seule la partie ouest du bâtiment 2 bénéficie de plusieurs rangs de pierres dont au moins un est destiné à être visible hors sol. D'importants blocs (dés) sont implantés régulièrement aux extrémités des solins, à leur intersection et plus ponctuellement au droit des portes, ce qui sous-entend la présence d'un pan de bois à sablière discontinue. Rien ne permet toutefois d'envisager plus sérieusement l'élévation des bâtisses et d'imaginer un pan de bois à cadres superposés ou à poteaux continus et bois longs. Chaque pièce du bâtiment 1 permet d'accéder directement par un jeu de portes aux extérieurs de l'édifice (au nord, puis à la venelle au sud). Il n'y a cependant aucun accès direct possible entre les différentes pièces du bâtiment. Si les deux principales pièces du bâtiment 2 adoptent les mêmes principes de distribution vers la venelle nord (accès direct et indépendant), elles communiquent chacune par un jeu de portes vers un vestibule distribuant lui-même sur la venelle sud et sur la tour d'angle (et l'éventuel étage associé). L'absence d'éléments lapidaires (jambage de portes ou encadrement de fenêtres) permet d'envisager des ouvertures confectionnées en matériaux périssables (bois). L'analyse architecturale des édifices permet d'envisager des toitures à deux pans symétriques dans le cas du bâtiment 1 et légèrement dissymétriques dans le cas du bâtiment 2 . La position centrale du faîtage est corroborée dans les deux cas par l'emplacement des cheminées dans les différentes pièces, ces dernières étant par commodité et technicité axées autant que possible sur l'axe du faîtage favorisant ainsi le tirage du feu et l'évacuation parfaite des fumées. L'étroitesse des bâtiments ainsi que l'absence de supports porteurs de la panne faîtière au sol permettent d'envisager une charpente à fermes pourvue d'entraits et d'arbalétriers. Ce type de charpente est par ailleurs plus approprié à des toits pentus, ce qui explique l'absence de tuiles sur le site, ces dernières étant plus adaptées à des toits à faible pente. Il est par conséquent plus que probable que les bâtiments de Chaillon soient couverts de matériaux périssables. Les sols sont majoritairement constitués de chapes de grouine chaulée et plus rarement de terre battue ou de petits cailloutis compactés et indurés reposant sur un radier de blocs calcaires. La découverte de quelques fragments de carreaux de terre cuite vernissés et décorés permet d'envisager ponctuellement et / ou localement un autre type de sol dont seuls subsisteraient quelques éléments épars. Le site de Bras-sur-Meuse a livré un édifice présentant de nombreuses similitudes architecturales et spatiales avec les bâtiments de Chaillon. 


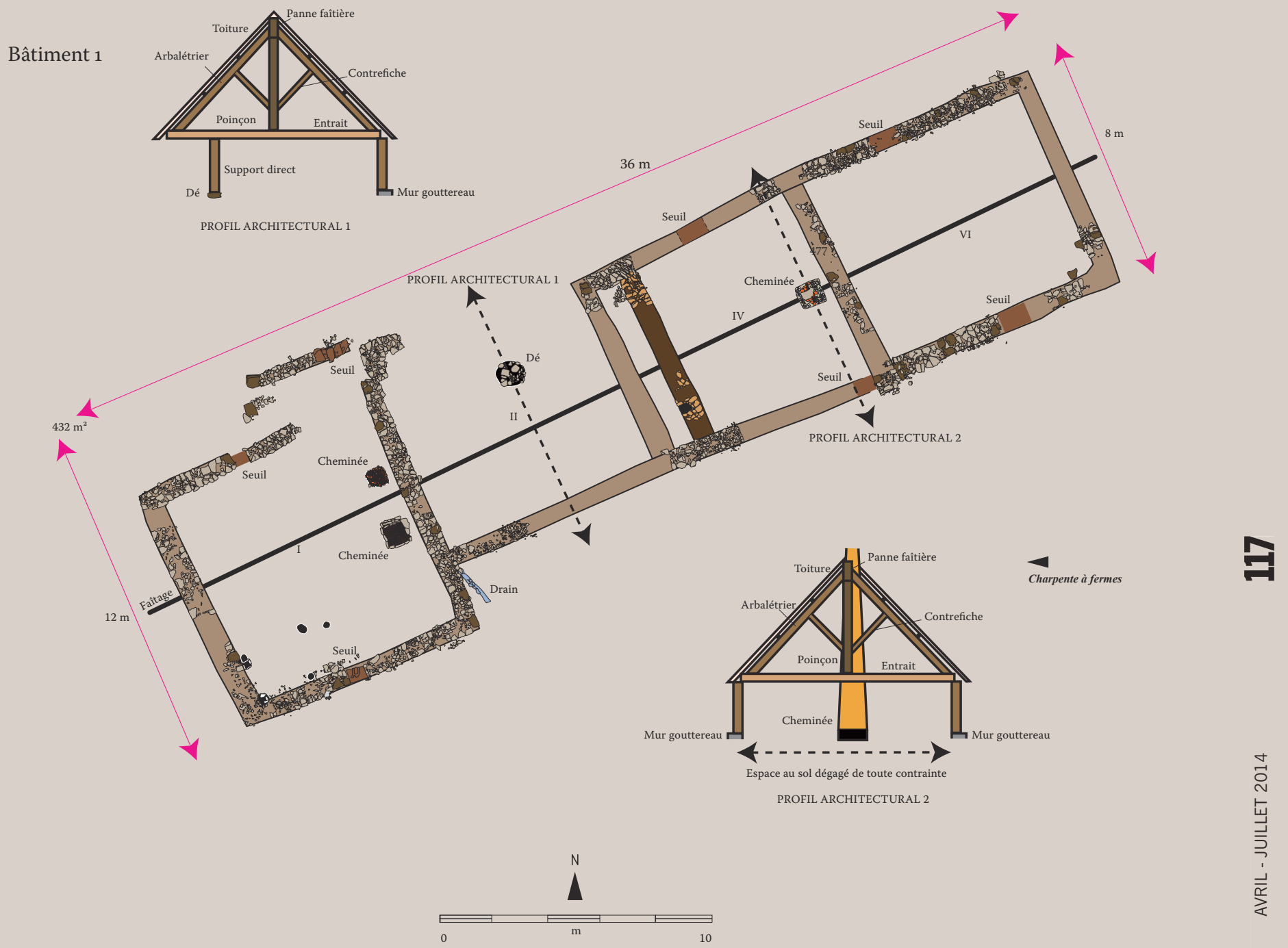

\section{Bâtiment 2}

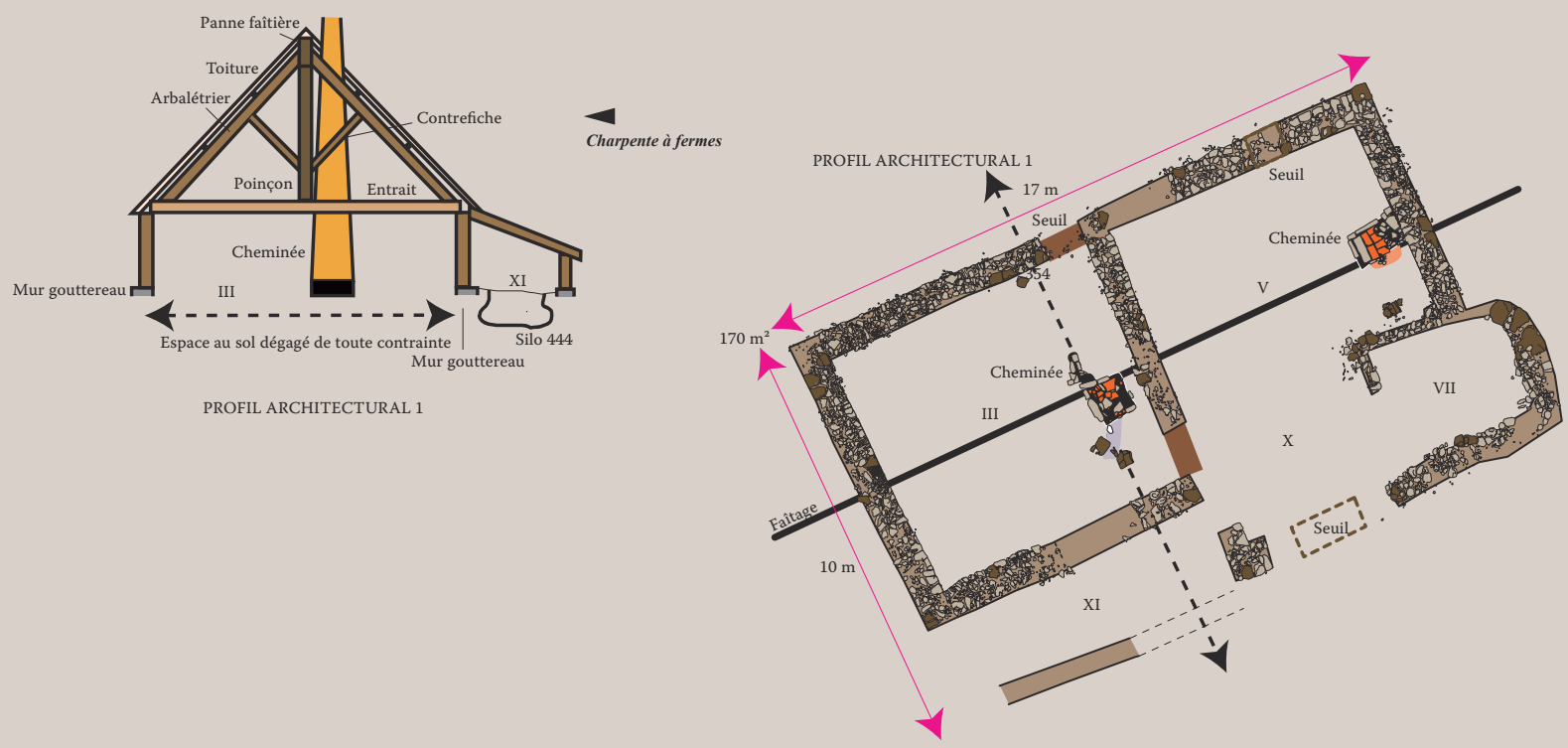


murs et façades sujets aux intempéries. Le développement d'un pan de bois sur un mur fondé en pierres haut de quelques mètres seulement est encore fréquemment observé en Moselle. Ce type d'élévation est d'ailleurs souvent représenté dans l'iconographie depuis la fin du xvie siècle. À l'exception de l'annexe (couverte de bois ou de matériaux périssables) flanquée contre le mur gouttereau est de l'édifice, l'ensemble du bâtiment dispose d'une toiture de tuiles canal à crochet en terre cuite. L'organisation interne du bâtiment ainsi que l'étude spatiale des supports de poteaux porteurs de la panne faîtière permettent d'envisager une toiture à deux pans dissymétriques (deux tiers est et un tiers ouest) et dont l'ossature générale intègre l'annexe latérale. Le logis est composé d'une pièce centrale et de deux pièces latérales. Si ces dernières sont généralement localisées dans l'alignement de la pièce centrale (l'une en façade et l'autre côté jardin), à Lucy, ces deux pièces sont situées côte à côte dans la largeur du logis. La pièce centrale (ou cuisine) constitue le lieu privilégié de la maison lorraine. Ici comme ailleurs, elle est un passage obligé pour accéder aux autres pièces. Flle regroupe tous les éléments autour desquels va s'organiser la vie familiale et occupe une superficie de plus de $92 \mathrm{~m}^{2}$. On y accède depuis l'extérieur par une porte installée dans le mur sud du logis. Si les bâtiments du bas Moyen Âge étudiés jusqu'ici en Lorraine disposaient tous de cheminées flanquées contre un des murs de la pièce de vie, à Lucy, le chauffage paraît assuré par un poêle disposé en position centrale dans la pièce. Au moins une fenêtre, attestée par la découverte de fragments de fenêtre rectangulaire à berceau en calcaire de Jaumont, devait permettre d'éclairer et d'aérer cette pièce. Quelques lampes à huile assuraient un éclairage plus direct. L'emplacement d'une pierre à eau est supposé dans l'angle sud-ouest de la pièce, non loin de la porte d'accès à l'extérieur. Si l'eau était évacuée directement par un drain traversant la pièce, il semblerait que son acheminement devait se faire manuellement depuis le puits. Aucune observation archéologique ne permet d'envisager la présence d'un étage. L'absence de cave et de four à pain est par ailleurs à noter. Une des particularités du schéma proposé à Lucy réside dans l'absence de corridor de distribution séparant les activités domestiques des activités agricoles.
On y passe de l'un à l'autre par une large porte. Les maisons lorraines plus récentes disposent toutes de ce couloir par lequel on pénètre dans l'édifice et depuis lequel on gagne l'une ou l'autre des travées d'habitation ou d'exploitation. Le sol semble être constitué de terre battue, à l'exception peut-être des emplacements de la pierre à eau et du poêle. Les pièces latérales ont une superficie de 15 à $17 \mathrm{~m}^{2}$. Dans les modèles de maisons lorraines plus récentes, ces chambres ou «poêles » sont généralement pourvues d'un parquet sur solives. La maison-bloc de Lucy se distingue par la présence de la seconde chambre aux côtés du poêle situé en façade. Elle est plus généralement orientée sur les jardins, à l'arrière de l'édifice dans le prolongement de la cuisine centrale. Le corps d'exploitation occupe une superficie de plus de $300 \mathrm{~m}^{2}$. Divisé d'ordinaire en deux travées caractérisées par la grange puis par les écuries et étables, à Lucy, le corps d'exploitation ne présente pas de divisions internes marquées. Seuls les supports au sol de la panne faîtière ainsi que quelques supports supplémentaires associés à un caniveau semblent coordonner cet espace. Ils délimitent un quadrilatère de près de $55 \mathrm{~m}^{2}$ assimilable à une zone de stabulation localisée dans l'angle nord-est du corps d'exploitation. Si les maisons lorraines " récentes » disposent d'une porte de grange, les modèles les plus anciens du bas Moyen Âge, étudiés grâce aux investigations archéologiques, n'ont jamais livré de vestiges s'apparentant à de telles ouvertures. Flles ne figurent par ailleurs jamais sur les représentations graphiques du xvr siècle. À Lucy pourtant, la présence d'une porte de grange n'est pas exclue dans l'axe de la zone de stabulation et au nord de l'annexe, située à l'arrière de la maison dans le prolongement du logis et le long du corps d'exploitation. Flle occupe une superficie totale de $72 \mathrm{~m}^{2}$. Si elle est intégrée à l'architecture générale de l'édifice, elle ne bénéficie pas pour autant de la même nature de couverture. Un espace cloisonné plus restreint (un peu plus de $25 \mathrm{~m}^{2}$ ) est localisé au sud de l'annexe dans le prolongement de la pièce principale du logis. Sa proximité avec la pièce chauffée ainsi que son accès direct depuis l'extérieur permettent d'envisager un lieu de stockage du bois de chauffe. soit sur le premier étage uniquement soit sur l'ensemble de la hauteur des murs, avec une priorité accordée aux 


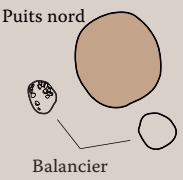

9
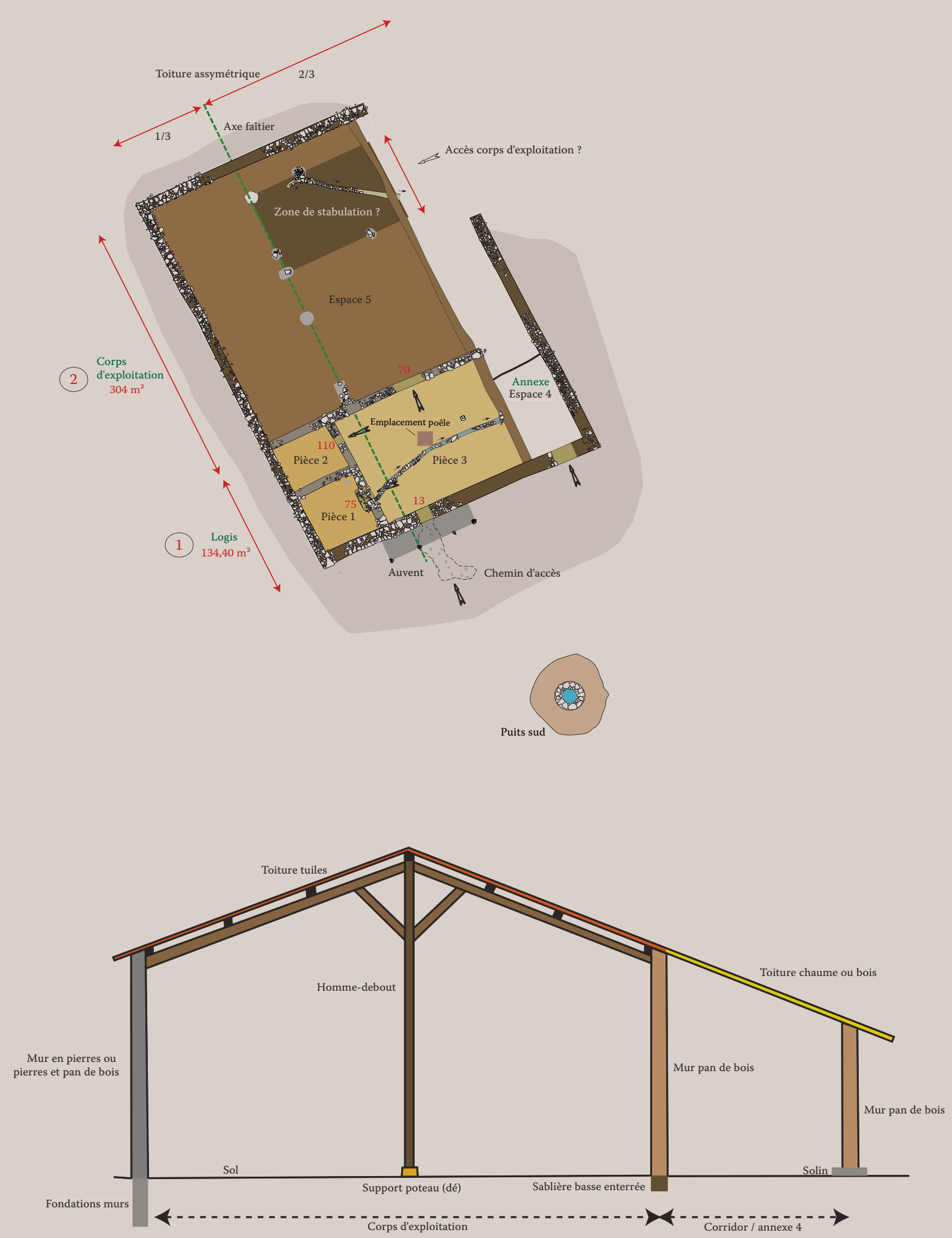